\title{
Peripheral vestibular dysfunction in patients with primary ciliary dyskinesia
}

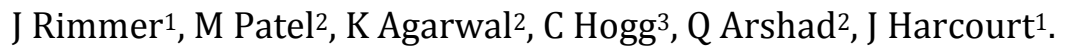 \\ 1 Department of Otolaryngology and Audiological Medicine, Charing Cross Hospital \\ ${ }^{2}$ Division of Brain Sciences, Imperial College London, Charing Cross Hospital \\ ${ }^{3}$ Department of Respiratory Medicine, Royal Brompton Hospital
}

Corresponding author: J Rimmer

\section{Introduction}

Primary ciliary dyskinesia (PCD) is a rare genetic defect of ciliary structure and/or motility (dyskinesia) with an incidence of approximately 1:15,000 [1]. Beating cilia located on the surfaces of cells of many tissues create localised fluid flow that regulates a plethora of developmental and physiological functions [2]. In PCD the defect is of motile cilia, found throughout the respiratory tract, including the Eustachian tubes. This results in the classical presentation of reduced mucociliary clearance from the upper and lower respiratory tract with subsequent chronic respiratory infections and eventual bronchiectasis. The most common otological symptom of PCD is conductive hearing loss associated with otitis media [1,3], indicating the important role cilia have in the middle ear. PCD does affect cilia in other regions of the body as well, for example the Fallopian tubes and the tails of spermatozoa with resultant subfertility. Situs inversus (reversal of the internal organs) occurs in almost 50\% of PCD patients, due to defective function of embryonic nodal cilia which leads to apparent randomisation of left-right axis development [4]. Patients with PCD may have associated ciliary defects such as retinitis pigmentosa; where such conditions co-exist patients are considered to have syndromic ciliopathies of which PCD is a distinct entity.

Within the inner ear, each chamber contains sensory epithelium comprising support and sensory hair cells [5]. On the apical surface of sensory hair cells are ciliary bundles which comprise a single microtubule-based kinocilium and numerous actin-based stereocilia [6]. In the human utricule and saccule, sensory epithelia are also associated with otoconia, which are involved in sensing gravity and linear head acceleration. Recent investigations in zebrafish suggest that ciliation is important for development of 
their otolith organ $[7,8]$; otolith formation, positioning or nucleation might be regulated by ciliated cells known as kinocilia (tether cells) [9]. These kinocilia may serve to distribute precursor particles and localise otolith deposition for seeding and positioning. The general hypothesis in humans is that kinocilium motility induces local fluid fluxes that propel otoconial precursor particles toward the base of the cilia, and in the direction of the growing stereocilia, ensuring that seeding particles are selectively drawn toward the anterior and posterior poles of the otic vesicle, and that otoconial formation is initiated only in these localized regions $[7,10]$. It remains unclear exactly where these cilia are located, but in zebrafish there are short motile and non-motile cilia throughout the otic vesicle and longer motile cilia at the poles $[7,11]$; the same may be true in humans although mammalian otoconial development is still not well understood.

As there are widespread effects on ciliary function in PCD, we speculate that the PCD defect might affect kinocilia structure and/or motility, and subsequent position and/or orientation of the otoconia. Since the position and orientation of otoconia are deeply involved in signal mechanotransduction from sensory hair cells, an altered position or orientation of otoconia could impair mechanical transference between the otoconia and sensory hair cells, impairing full function from the saccule and utricle. With the important role of ciliation for otolith development in zebrafish, we investigated whether PCD, a congenital disorder of ciliary function, is associated with impaired human otoconial organ function, using routine clinical tests. The present pilot study is the first to assess vestibular function in PCD. We observed impaired saccular and utricular function in this patient cohort.

\section{Materials and Methods}

Ethical approval was sought and obtained from the National Research Ethics Service via the Joint Research Office at Imperial College Healthcare NHS Trust and the North London Research Ethics Committee 2 (protocol ID: JROHH0046; REC reference 10/H0701/96; ClinicalTrials.gov ID: NCT01246258). All procedures followed were in accordance with the ethical standards of the National Research Ethics Service, the NHS Research Governance Framework for Health and Social Care (2nd edition), and with the Helsinki Declaration.

\section{Subjects}

Table 1 shows the ages and phenotype for each patient, based on nasal brushings. Figure 1a shows the abnormal cilia found in nasal brushings from subject 4, with 
absent/abnormal outer dynein arms, compared to a normal cilium (Figure 1b). Subject 5's normal electron microscopy (EM) appearances with dyskinetic ciliary movement are consistent with his known genetic mutation (DNAH11).

\section{Audiometry}

Hearing tests were air conduction, pure-tone audiometry thresholds at $0.25,0.5,1,2,3$, 4, 6 and $8 \mathrm{kHz}$. Subject 2 was not tested at 3 and $6 \mathrm{kHz}$. Headphones were used within a soundproof booth. An average from all thresholds was calculated for both ears.

\section{Tympanometry}

Tympanometry captured acoustic impedance from -400 to 200 daPa using a small tip inserted into the ear canal. Each ear was tested once during which participants were asked not to swallow or speak, yielding the peak pressure of tympanometric compliance and middle ear volume.

\section{Cervical Vestibular Evoked Myogenic Potential (cVEMP)}

A custom system (Faldon \& Buckwell) simultaneously delivered air-conducted sound monaurally via audiological headphones and captured electromyographic (EMG) activity. Sound was delivered as $500 \mathrm{~Hz}$ clicks at $110 \mathrm{~dB}$ nHL (120dB SPL) of positive polarity, repeating 5 times per second. EMG signals were averaged over two hundred sweeps and rectified. Prior to electrode (Ag/AgCl, W-60, Skintact UK) adhesion, skin was cleaned (NuPrep, Weaver Company, USA) and electrodes gelled (Spectra 360, Parker, USA). Resistance across electrodes was $<5 \mathrm{~K} \Omega$. The electrode montage comprised the right and left sternocleidomastoid (SCM) muscle bellies, the right and left ipsilateral mid-clavical bones (reference) and sternum (ground).

Subjects lay on an examination bed, upper torso elevated with the backrest at $30^{\circ}$ and lower body flat, perpendicular to the ground. For testing, they were asked to elevate their heads from the head rest and turn either to the left (right ear test) or to the right (left ear test) to increase tonic neck muscle activity. A biofeedback device (NeuroTrac Simplex unit, NeuroTrac, UK) regulated contractional strength (approximately $30 \mu \mathrm{V}$ ). The 13 was taken as the first distinctive peak in waveform and $n 23$ as the first trough. VEMPs were scored in terms of presence or absence, p13 and n23 latency and percentage amplitude asymmetry.

Amplitude L - Amplitude R

VEMP asymmetry $=100 \times \quad$ Amplitude L + Amplitude R 


\section{Utricular Centrifugation (UCF)}

A full explanation of the protocol and theory for utricular centrifugation (UCF) has been published elsewhere [12-14]. In brief, utricular function was measured by the subjective visual vertical (SVV) during unilateral centrifugation [15]. Testing was performed with a vertical axis rotation chair (Neurokinetics Inc, Pittsburugh, USA) capable of lateral chair translation for eccentric displacement, in total darkness and with the subject's head, torso, legs and feet secured into the chair. Vertical head alignment was ensured with a vertically calibrated laser. Attached to the chair were two arms holding a thin wooden plaque $1 \mathrm{~m}$ from participants, onto which a laser projected an illuminated red dot or line $(160 \mathrm{~mm} \times 3 \mathrm{~mm})$. The line could be rotated about its centre using a wheel joy-pad given to subjects who were instructed to position the line to their gravitational vertical. Upon doing so, the patient was asked to give a vocal cue that the line was vertical. The line angle was saved, and the line re-set to a new angle. This was repeated over a 30 second period until the chair changed translation or after 7 SVV measurements. SVV was measured in the stationary position, during rotation centred on axis and during the left and right translations.

The UCF protocol was:

1. Chair stationary. SVV measured 7 times.

2. Chair accelerated at $3^{\circ} / \mathrm{s}^{2}$ up to a constant velocity of $400^{\circ} / \mathrm{s}$ over $120 \mathrm{~s}$ and maintained in the on-axis position for 60 s.

3. Translation from centre to the right laterally $(4 \mathrm{~cm}, 0.2 \mathrm{~cm} / \mathrm{s})$ over $30 \mathrm{~s}$ before SVV was measured for 30 s.

4. Translation back to centre over 30s, and SVV measured for 30s.

5. Translation from centre to the left laterally $(4 \mathrm{~cm}, 0.2 \mathrm{~cm} / \mathrm{s})$ over $30 \mathrm{~s}$, and SVV measured for $30 \mathrm{~s}$.

6. Translation back to the central position for $30 \mathrm{~s}$ and decelerated at $2.5^{\circ} / \mathrm{s}$ to rest.

During earth-centred centrifugation, displacing the head by $4 \mathrm{~cm}$ from the rotation axis stimulates the eccentrically positioned utricle unilaterally through the resultant centrifugal force. The resulting utriculo-ocular reflex (UOR) causes ocular counter-roll in an opposite direction to the translation direction. At $400^{\circ} / \mathrm{s}$ the stimulated utricle is exposed to a centrifugal acceleration of $\omega^{2} r=0.40 \mathrm{~g}$, where $\omega$ is the angular velocity and $r$ the distance between the axis of rotation and the stimulated utricle, giving rise to a tilt 
sensation of $11.2^{\circ}$ [14]. UCF was scored as median SVV angle and gain in each position and utricular percentage weakness. The normal range for static SVV is about $2^{\circ}[13,16]$.

$$
\text { UCF weakness }=200 \times \frac{\text { Median L }+ \text { Median C }+ \text { Median R }-3 \times \text { Median Static }}{3 \times(\text { Median L }- \text { Median } R)}
$$

\section{Electronystagmography (ENG)}

Semi-circular canal function was assessed by $90 \%$ s step rotations (right- and leftward) in terms of gain (ratio of eye velocity to head velocity) and time constant (time taken for eye movement velocity to decay to $36.8 \%$ of the maximum velocity). The time constant is a technique based on eye movement response to an acceleration impulse generated by a cessation of rotational chair power following an epoch of constant velocity. The vestibulo-ocular reflex was also measured by sinusoidal rotations at $0.25 \mathrm{~Hz}$. Eye movements were captured with three small adhesive electrodes (Carefusion, $\mathrm{Ag} / \mathrm{AgCl}$ ) stuck to the outer canthi of both eyes and forehead.

Testing was performed in the same rotation chair as the UCF (Neurokinetics Inc, Pittsburgh, USA) and again in complete darkness. No visual cues were present.

\section{Subjective balance function and symptoms}

The Vertigo Symptom Scale-short form (VSS) and Dizziness Handicap Inventory [17], both validated questionnaires, were completed by all subjects.

\section{Results}

Some subjects showed evidence of vestibular abnormality (Table 2).

\section{Pure-Tone Audiometry}

As seen in Figure 2, a unilateral mild/moderate conductive hearing loss was present in 4 of the 5 subjects. The remaining subject (subject 5) had mild bilateral conductive hearing loss.

\section{Tympanometry}

Table 3 shows that the stapedial reflex was reduced though not entirely eliminated. Tympanometry was available for all apart from subject 2. Otoscopic examination of this subject was unremarkable. 


\section{Vestibular Evoked Myogenic Potentials}

There were markedly reduced or unobtainable cVEMPs bilaterally in 3 of the 5 subjects $(1,2$, and 4$)$ and unilaterally in the remaining 2 subjects (Figure 3$)$. The amplitude asymmetry in subject 3 was significant at $71.9 \%$, but not in subject 5 (8.0\%), probably owing to a small VEMP amplitude.

\section{Utricular Centrifugation}

No subject had a pathological UCF asymmetry (i.e. $>100 \%$ weakness). Tilt perception was reduced unilaterally in subject 3 during left chair translation, as shown in Figure 4 by the closeness of the median tilt response to the dashed baseline mark. Subjects 1 and 4 showed small SVV responses bilaterally, represented by small gain values in table 2 .

\section{Electronystagmography}

The vestibulo-ocular reflex (VOR) at $0.25 \mathrm{~Hz}$ sinusoidal rotation was normal in all subjects. The rotational nystagmic response of subject 3 was processed differently. Nystagmic decay in this participant was normal in both rotational directions. Rotation to the right yielded a time constant of $9 \mathrm{~s}$ and to the left, 10s. Rotational time constants were borderline reduced bi-directionally in subjects 1 and 4; both had unattainable VEMPs bilaterally. Subject 5 had a borderline reduced time constant on the right side, the same side as his unattainable VEMP. Rotational time constants were normal in subjects 2 and 3. Gains were typical.

\section{Subjective balance function and symptoms}

No evidence of vertigo or abnormal dizziness was found in any subject, as shown by entirely normal questionnaire scores.

\section{Discussion}

The goal of this study was to assess whether PCD, a congenital disorder of ciliary function, impairs otoconial organ function expressed through routine vestibular clinical tests. We show that saccular and utricular function is impaired in this small cohort of PCD patients. This finding suggests that ciliary structure and/or motility could contribute to otoconial organ function by impairing otoconial position or development as previous studies in zebrafish suggest $[7,9]$. 
Congenital ciliary dysfunction in PCD commonly causes chronic respiratory infections and conductive hearing loss as result of otitis media. All subjects presented with a mild to moderate conductive hearing loss, but not the sensorineural loss that might be expected if motile cilia are required for normal development of the cochlea. A conductive hearing loss is frequently seen in PCD, commonly associated with otitis media with effusion [1]. In keeping with this, subject 3 had reduced tympanometric response and all except subject 4 had small middle ear volumes. Plausibly, hair cell function within the cochlea is preserved in PCD. We also observed an intact VOR in response to angular rotation (rVOR) in all 5 PCD subjects, confirming the presence of bilateral vestibular function. Upon rotational testing, however, the VOR time constant (which reflects vestibulo-central processing [18] and is typically 13-16s long [19]) was borderline reduced in subjects 1,4 and 5 (Table 2), similar to that seen in vestibular disorders (such as acute vestibular neuritis [20]) and motion-habituated professionals [21]. Perhaps the slight reduction in semicircular canal function points towards reciprocal inhibition to manage the otoconia-semicircular canal mismatch $[22,23]$.

Utricular function can be assessed by means of centrifugation (UCF) [23]. UCF has demonstrated utricular dysfunction in benign paroxysmal positional vertigo (BPPV) patients [24] and following intratympanic gentamicin therapy (causing ototoxic destruction of vestibular stereocilia and kinocilia) in 60-70\% of Meniere's disease patients [25]. Subjects 1, 3 and 4 showed utricular abnormalities, subject 3 unilaterally on the left and subjects 1 and 4 with bilaterally reduced gain (below 0.3 ). In comparison, the gain in the 20 healthy subjects presented in Figure 4 was 0.7 .

The cVEMP is an established test of saccular function [26]. Unobtainable or significantly reduced cVEMP responses were observed in all subjects. However, the conductive hearing loss present in these patients decreases cVEMP reliability [27] which may have affected our results. However, the conductive hearing loss seen in our patients was small or insignificant (Figure 2) which argues against unreliability. Still, bone-conducted cVEMP as opposed to air-conducted could provide better reliability [28].

Otoconial function, as measured by UCF (utricles) and VEMP (saccules), showed reduced function in this cohort of PCD patients compared to normal controls. The otoconial organs, arranged in the horizontal plane (utricle) and vertical plane (saccule), respond to linear acceleration and gravity, serving a functional role in the maintenance of posture via the vestibulospinal reflex. However, despite otoconial abnormalities the test 
subjects experienced no obvious postural impairment. Recently, unilateral ablation of vestibular function in tadpoles, resulting in disequilibrium in descending vestibulospinal information, led to an altered assembly of adult spinal locomotor circuitry and normal swimming behaviour after metamorphosis, in contrast to frogs lesioned after metamorphosis who never recovered [29]. These findings suggest adaptive compensation for vestibular dysfunction in the developmental stages. Similarly, subjects with chronic congenital nystagmus or chronic progressive external ophthalmoplegia are typically symptom free [30].

\section{Limitations}

A major factor worthy of discussion is our rotational protocol. We have chosen to use a step rotation at $90^{\circ} \mathrm{s}$ to maintain consistency with our previous studies $[20,31]$ and others of the velocity storage mechanism [32]. One measure of the velocity storage system is the vestibular velocity storage mechanism. We have chosen this measure because the time constant can reflect vestibular impairment by disease $[20,33]$, intervention [31] and drug effects [21,34-36]. However, it should be pointed out that the time constant can habituate which lends itself to poor repeatability. The tests were only performed once for rightward and leftward rotations to prevent a habituation effect. Furthermore, sinusoidal rotation at $0.25 \mathrm{~Hz}$ is limited compared to the range of frequencies available where the phase could produce a more reliable measure of canal dysfunction than gain. Importantly though, we showed larger human abnormalities in otoconial function than semicircular canal function in PCD, which is consistent with previous theories on ciliary motility and otolith seeding, positioning [9] in zebrafish.

\section{Conclusion}

We speculate that the reduced saccular and utricular function in PCD patients observed in this pilot study suggests a relationship between this rare congenital disease, which affects cilia structure and/or motility, and the otoconia seeding and/or positioning. The PCD patients did not report any subjective dizziness or vertigo, suggesting subclinical vestibular dysfunction. However, this was a small pilot study and further investigation of a larger cohort of PCD patients is warranted. 


\section{References}

1. Rimmer J. Congenital problems of mucociliary clearance: primary ciliary dyskinesia. Rhinology 2012;50:353-9.

2. Roy S. The motile cilium in development and disease: emerging new insights. Bioessays 2009;31:694-9.

3. Badano JL, Mitsuma N, Beales PL, Katsanis N. The ciliopathies: An emerging class of human genetic disorders. Annu Rev Genom Hum Genet 2006;7:125-8.

4. Castleman VH, Romio L, Chodhari R, et al. Mutations in radial spoke head protein genes RSPH9 and RSPH4A cause primary ciliary dyskinesia with centralmicrotubular-pair abnormalities. Am J Hum Genet 2009;84:197-209.

5. Anniko M. Cytodifferentiation of cochlear hair cells. Am J Otolaryngol 1983;4:375-88.

6. Bisgrove BW, Yost HJ. The roles of cilia in developmental disorders and disease. Development 2006;133:4131-43.

7. Colantonio JR, Vermot J, Wu D, et al. The dynein regulatory complex is required for ciliary motility and otolith biogenesis in the inner ear. Nature 2009;457:2059.

8. Riley BB, Zhu C, Janetopoulos C, Aufderheide KJ. A critical period of ear development controlled by distinct populations of ciliated cells in the zebrafish. Dev Biol 1997;191:191-201.

9. Stooke-Vaughan GA, Huang P, Hammond KL, Schier AF, Whitfield TT. The role of hair cells, cilia and ciliary motility in otolith formation in the zebrafish otic vesicle. Development 2012;139:1777-87.

10. Wirschell $\mathrm{M}$, Olbrich $\mathrm{H}$, Werner $\mathrm{C}$, et al. The nexin-dynein regulatory complex subunit DRC1 is essential for motile cilia function in algae and humans. Nat Genet 2013;45:262-8. 
11. Wu D, Freund JB, Fraser SE, Vermot J. Mechanistic basis of otolith formation during teleost inner ear development. Dev Cell 2011;20:271-8.

12. Clarke AH, Schonfeld U, Helling K. Unilateral examination of utricle and saccule function. J Vestib Res 2003;13:215-25.

13. Akin FW, Murnane OD, Pearson A, Byrd S, Kelly KJ. Normative data for the subjective visual vertical test during centrifugation. J Am Acad Audiol 2011;22:460-8.

14. Weerts AP, De Meyer G, Pauwels G, et al. Pharmaceutical countermeasures have opposite effects on the utricles and semicircular canals in man. Audiol Neurootol 2012;17:235-42.

15. Janky KL, Shepard NT. Unilateral centrifugation: utricular assessment and protocol comparison. Otol Neurotol 2011;32:116-21.

16. Janssen M, Lauvenberg M, van der Ven W, Bloebaum T, Kingma H. Perception threshold for tilt. Otol Neurotol 2011;32:818-25.

17. Morris AE, Lutman ME, Yardley L. Measuring outcome from vestibular rehabilitation, part II: refinement and validation of a new self-report measure. Int J Audiol 2009;48:24-37.

18. Ramat S, Bertolini G. Estimating the time constants of the rVOR. A model-based study. Ann N Y Acad Sci 2009;1164:140-6.

19. Raphan T, Matsuo V, Cohen B. Velocity storage in the vestibulo-ocular reflex arc (VOR). Exp Brain Res 1979;35:229-48.

20. Cousins S, Kaski D, Cutfield N, et al. Vestibular perception following acute unilateral vestibular lesions. PLoS One 2013;8:e61862.

21. Cohen B, Dai M, Raphan T. The critical role of velocity storage in production of motion sickness. Ann N Y Acad Sci 2003;1004:359-76. 
22. Deutschlander A, Bense S, Stephan T, Schwaiger M, Brandt T, Dieterich M. Sensory system interactions during simultaneous vestibular and visual stimulation in PET. Hum Brain Mapp 2002;16:92-103.

23. Schonfeld U, Clarke AH. A clinical study of the subjective visual vertical during unilateral centrifugation and static tilt. Acta Otolaryngol 2011;131:1040-50.

24. von Brevern M, Schmidt T, Schonfeld U, Lempert T, Clarke AH. Utricular dysfunction in patients with benign paroxysmal positional vertigo. Otol Neurotol 2006;27:92-6.

25. Helling K, Schonfeld U, Clarke AH. Treatment of Meniere's disease by low-dosage intratympanic gentamicin application: effect on otolith function. Laryngoscope 2007;117:2244-50.

26. Colebatch JG, Halmagyi GM, Skuse NF. Myogenic potentials generated by a clickevoked vestibulocollic reflex. J Neurol Neurosurg Psychiatry 1994;57:190-7.

27. Zhou G, Poe D, Gopen Q. Clinical use of vestibular evoked myogenic potentials in the evaluation of patients with air-bone gaps. Otol Neurotol 2012;33:1368-74.

28. Yang TL, Young YH. Comparison of tone burst and tapping evocation of myogenic potentials in patients with chronic otitis media. Ear Hear 2003;24:191-4.

29. Beyeler A, Rao G, Ladepeche L, Jacques A, Simmers J, Le Ray D. Vestibular lesioninduced developmental plasticity in spinal locomotor networks during Xenopus laevis metamorphosis. PLoS One 2013;8:e71013.

30. Seemungal BM, Masaoutis P, Green DA, Plant GT, Bronstein AM. Symptomatic recovery in Miller Fisher syndrome parallels vestibular-perceptual and not vestibular-ocular reflex function. Front Neurol 2011;2:2.

31. Arshad Q, Nigmatullina Y, Bronstein AM. Handedness-related cortical modulation of the vestibular-ocular reflex. J Neurosci 2013;33:3221-7. 
32. Bertolini G, Ramat S, Laurens J, et al. Velocity storage contribution to vestibular self-motion perception in healthy human subjects. J Neurophysiol 2011;105:20923.

33. Okada T, Grunfeld E, Shallo-Hoffmann J, Bronstein AM. Vestibular perception of angular velocity in normal subjects and in patients with congenital nystagmus. Brain 1999;122:1293-303.

34. Cohen B, Dai M, Yakushin SB, Raphan T. Baclofen, motion sickness susceptibility and the neural basis for velocity storage. Prog Brain Res 2008;171:543-53.

35. Shaikh AG, Marti S, Tarnutzer AA, et al. Effects of 4-aminopyridine on nystagmus and vestibulo-ocular reflex in ataxia-telangiectasia. J Neurol 2013;260:2728-35.

36. Shaikh AG, Palla A, Marti S, et al. Role of cerebellum in motion perception and vestibulo-ocular reflex-similarities and disparities. Cerebellum 2013;12:97-107. 


\section{Figures}

A

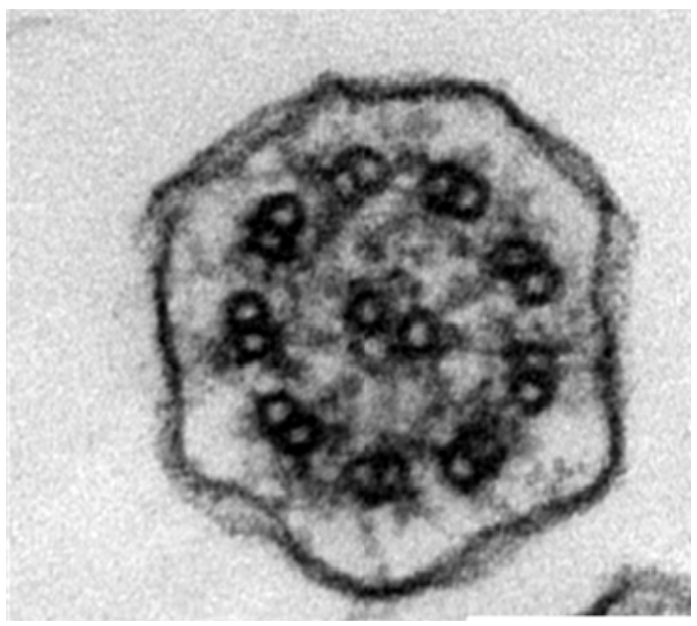

B

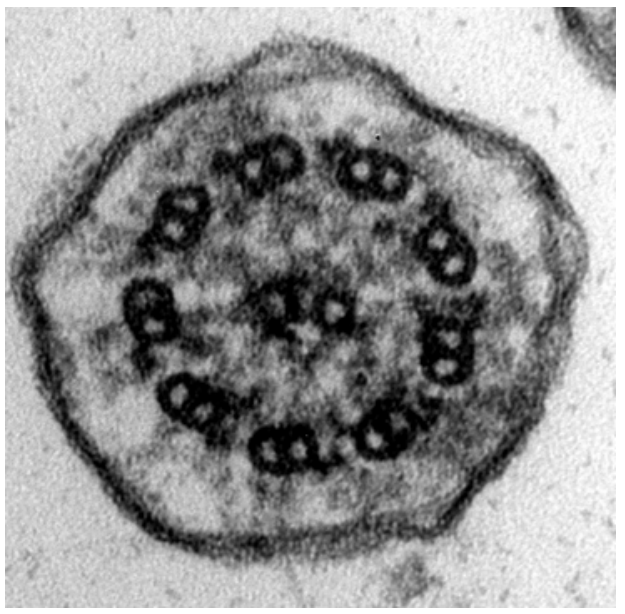

Figure 1a: Electron micrograph showing transverse section of a cilium lacking outer dynein arms (absence indicated by red arrows); where present outer dynein arms appear shortened (x 100,000) Figure 1b: Electron micrograph showing transverse section of a normal cilium (x 100,000) 

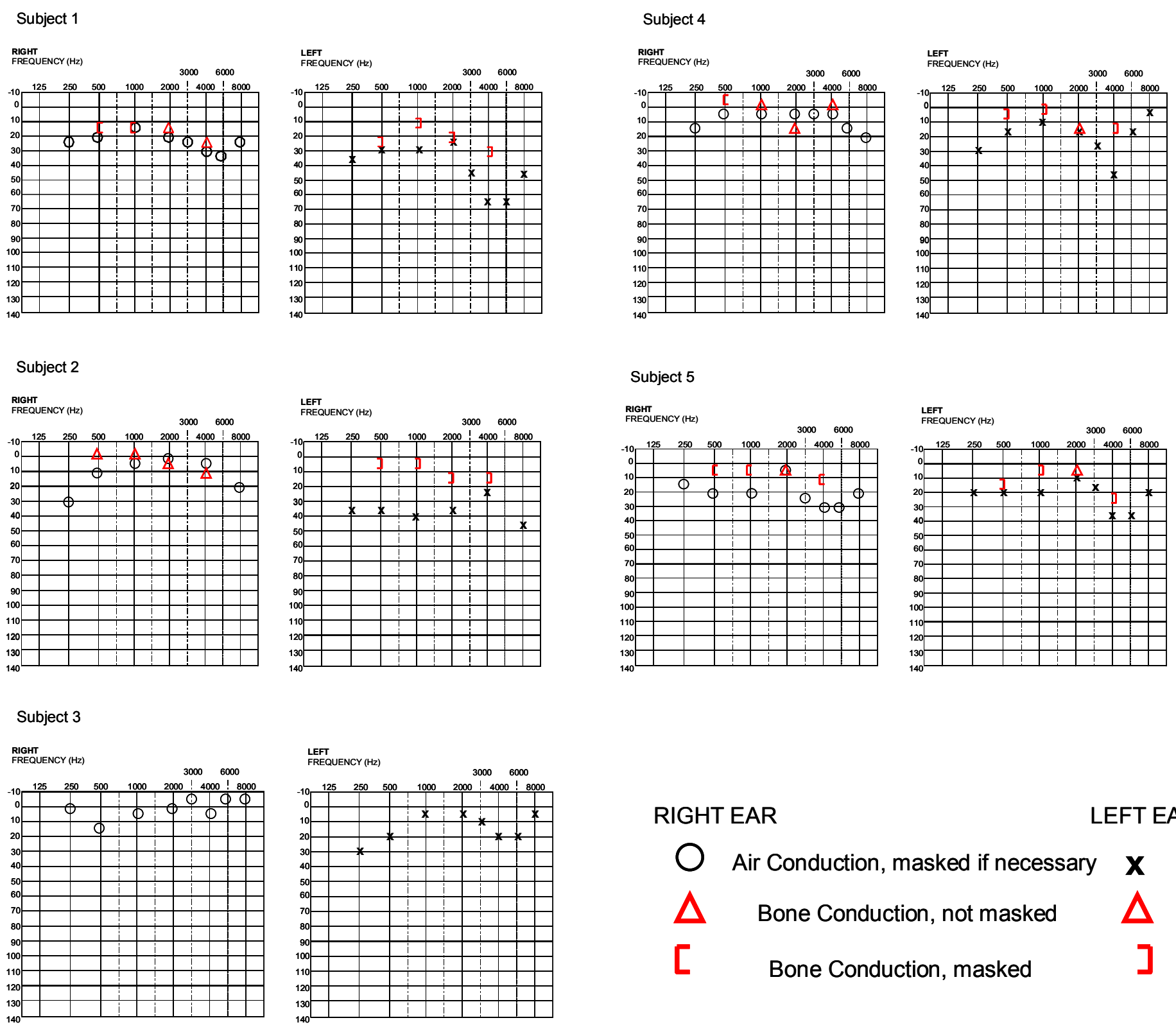

Figure 2: Pure-tone audiograms from the five PCD subjects 

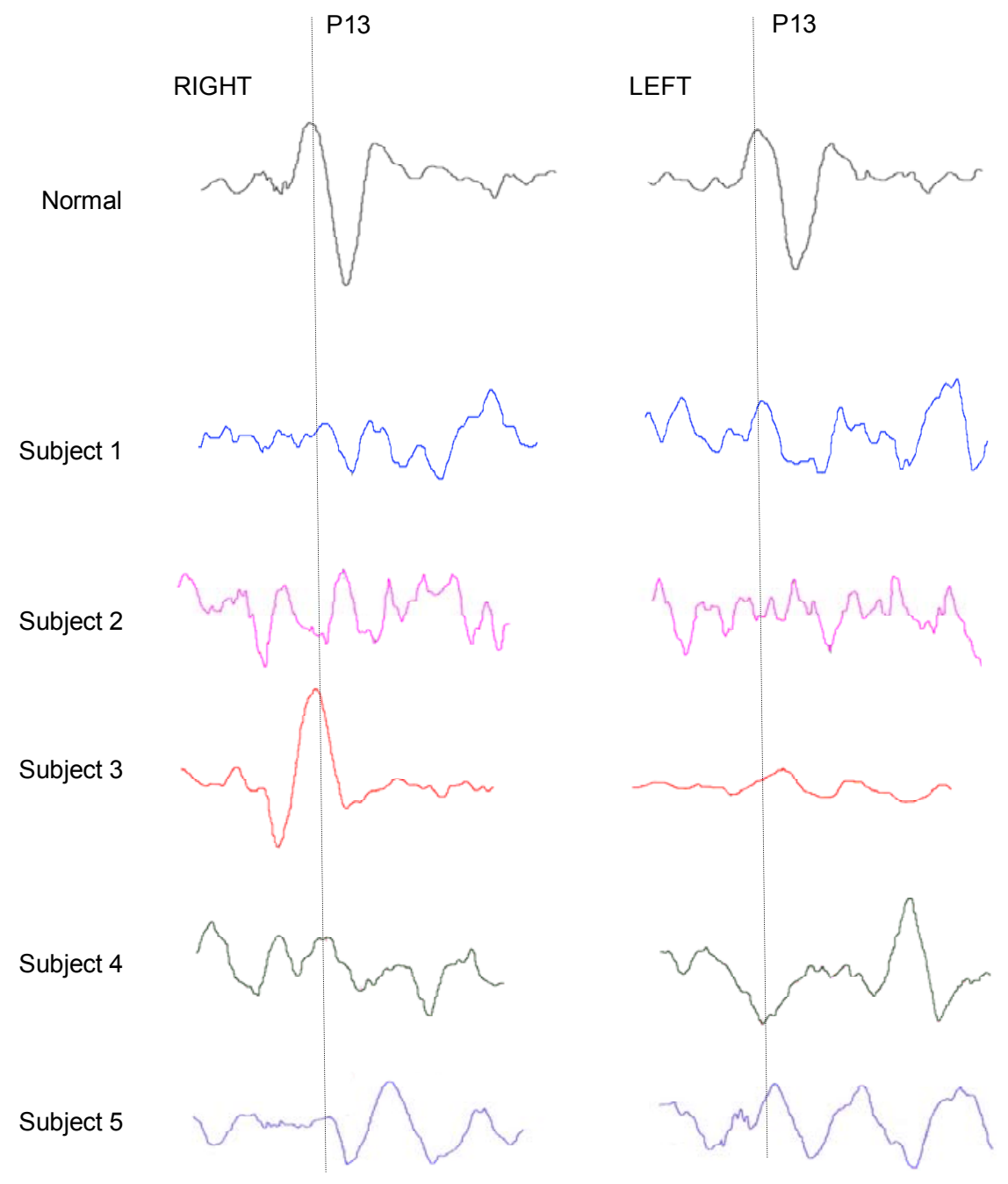

Figure 3: VEMPs of the 5 PCD subjects and of a healthy normal subject. Reductions in VEMP responses are clearly visible. Only the right VEMP in subject 3 is in keeping with a normal response. 


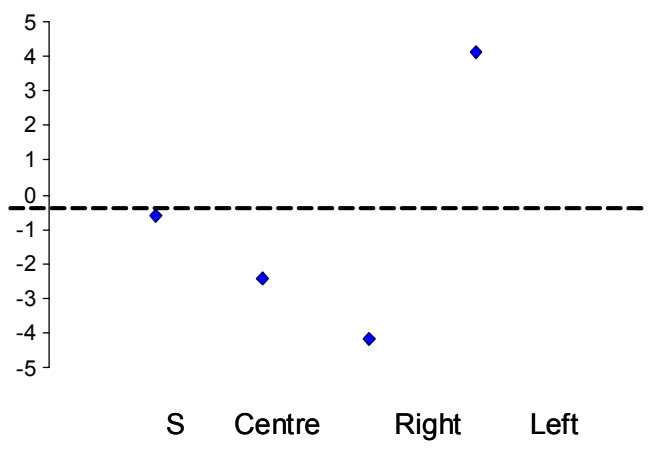

Subject 2

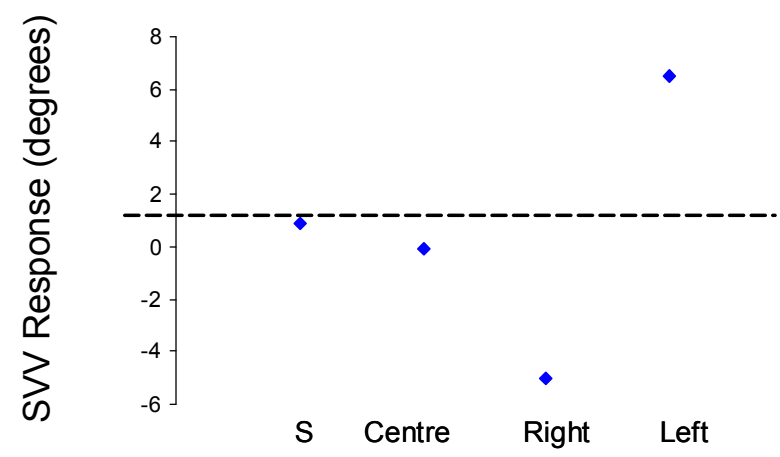

Subject 3

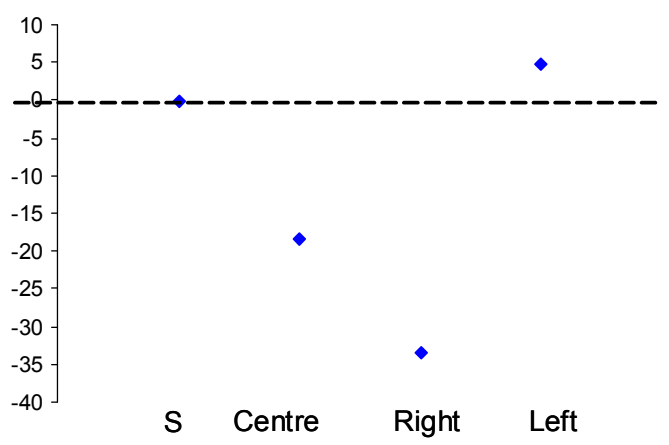

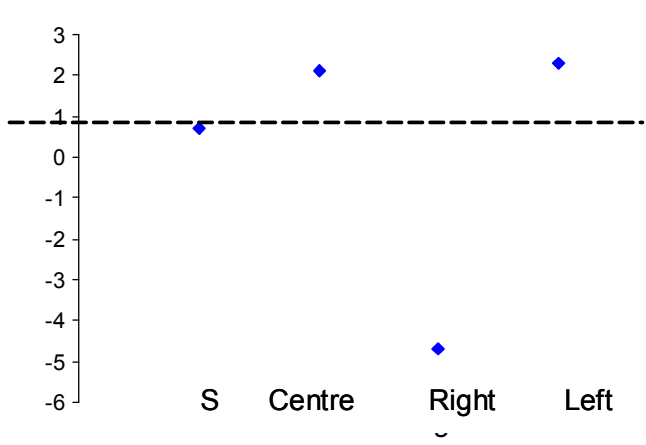

Subject 5

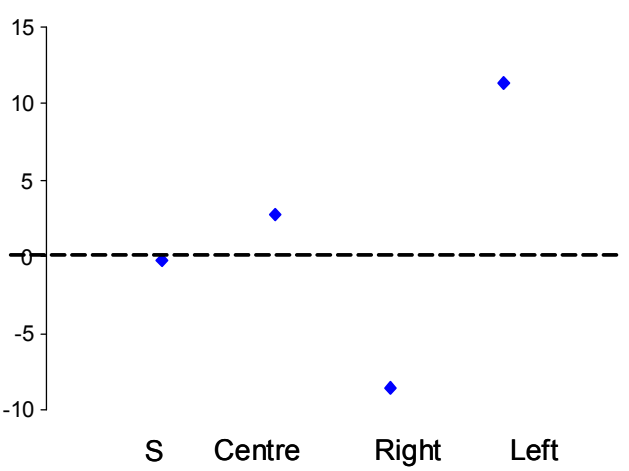

Healthy group

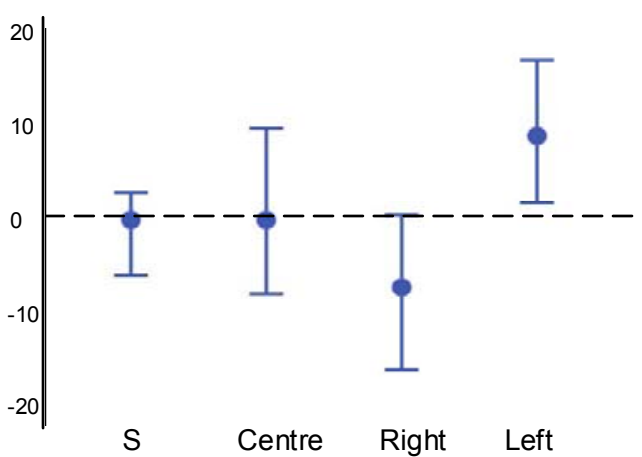

Figure 4: Median UCF SVV responses (degrees) to stationary (S), centre centrifugation and right and left chair translations in all five PCD subjects and a healthy group (20 control subjects, minimum-maximum range given by the bar and mean (circle)). The dashed line indicates the subject's static vertical perception. Subject 3 showed evidence of reduced tilt perception on left chair translation. 


\section{Tables}

\begin{tabular}{|l|l|l|l|l|l|}
\hline Patient & Age & Ciliary function (LM) & Defect (EM) & Symptoms & Situs \\
\hline 1 & 28 & Unknown & Unknown & $\begin{array}{l}\text { URT } \\
\text { LRT }\end{array}$ & Normal \\
\hline 2 & 28 & Static & ODA & $\begin{array}{l}\text { URT } \\
\text { LRT }\end{array}$ & Normal \\
\hline 3 & 29 & Unknown & Unknown & $\begin{array}{l}\text { URT } \\
\text { LRT }\end{array}$ & Inversus \\
\hline 4 & 18 & Static & ODA & $\begin{array}{l}\text { URT } \\
\text { LRT }\end{array}$ & Normal \\
\hline 5 & 18 & $\begin{array}{l}\text { Dyskinetic with } \\
\text { reduced beat frequency }\end{array}$ & Normal EM & $\begin{array}{l}\text { URT } \\
\text { LRT }\end{array}$ & Inversus \\
\hline
\end{tabular}

Table 1: Patient details (LM=light microscopy, EM=electron microscopy,

$\mathrm{ODA}=$ outer dynein arm, URT=upper respiratory tract, LRT=lower respiratory tract)

\begin{tabular}{|l|l|l|l|l|l|l|l|l|l|l|}
\hline Subject & \multicolumn{9}{l}{ PTA (dB) } & \multicolumn{2}{l|}{ VEMP } & \multicolumn{2}{l|}{ UCF } & \multicolumn{2}{l|}{ ENG } \\
\hline & R & L & R & L & & & R & L & \\
\hline & & & & & Gain & Asym & Gain & TC & Gain & TC \\
\hline $\mathbf{1}$ & 24.4 & 42.5 & A & A & 0.36 & $6-$ L & 0.76 & 5 & 0.67 & 5.2 \\
\hline $\mathbf{2}$ & 11.6 & 36.7 & A & A & 0.49 & $7-$ L & 0.45 & 14.2 & 0.4 & 10.7 \\
\hline $\mathbf{3}$ & 1.25 & 16.9 & P & A & 1.64 & $81-$ L & N/A & 9 & N/A & 10 \\
\hline $\mathbf{4}$ & 9.4 & 20 & A & A & 0.30 & $23-$ L & 0.55 & 5.9 & 0.49 & 3.6 \\
\hline $\mathbf{5}$ & 20.6 & 21.9 & P & A & 0.85 & $20-\mathrm{R}$ & 0.56 & 6.7 & 0.47 & 12.4 \\
\hline
\end{tabular}

Table 2: Pure tone thresholds (averaged across all frequencies), VEMP, UCF and ENG results in all participants. $(\mathrm{A}=$ absent, $\mathrm{P}=$ present, $\mathrm{L}=$ left, $\mathrm{R}=$ right, Asym=asymmetry, $\mathrm{TC}=$ time constant, $\mathrm{N} / \mathrm{A}=$ not available) 


\begin{tabular}{|l|l|l|l|l|}
\hline Subject & \multicolumn{3}{|l|}{ Right } & Left \\
\hline & Peak & Vol (ml) & Peak & Vol (ml) \\
\hline $\mathbf{1}$ & -340 & 0.3 & -190 & 0.1 \\
\hline $\mathbf{2}$ & N/A & & N/A & \\
\hline $\mathbf{3}$ & 5 & 0.2 & 5 & 0.2 \\
\hline $\mathbf{4}$ & -35 & 0.8 & -40 & 0.8 \\
\hline $\mathbf{5}$ & -75 & 0.1 & -90 & 0.2 \\
\hline
\end{tabular}

Table 3: Tympanometric results from the five PCD subjects 